\title{
Adolescents and young adults on the acute medical unit: how might we do it better?
}

\author{
Authors: Lorraine Albon ${ }^{A}$ and Louella Vaughan ${ }^{B}$
}

\section{It is a common perception that young people do not become ill and do not pose a challenge in the unscheduled healthcare setting. The research, however, increasingly suggests that young adults and adolescents (YAAs) are a highly vulnerable group, with poorer outcomes than either older adults or children, and distinct healthcare needs. The acute medical unit (AMU) setting poses particular challenges to the care of this patient group. To improve care and patient experience, adult clinicians need to look critically at their services and seek to adapt them to meet the needs of YAAs. This requires cooperation and linkage with local paediatric and emergency services, as well as the input of other relevant stakeholder groups. Staff on AMUs also need to develop the knowledge, skills and attitudes to communicate effectively and address the developmental and health needs of YAAs and their parents/carers at times of high risk and stress.}

KEYWORDS: Adolescents, young adults, age-appropriate care, acute medical units

\section{Introduction}

It is a common perception that young people do not become ill, consume only a small fraction of healthcare resources and do not pose a particular problem in unscheduled healthcare settings. ${ }^{1}$ The reality is that the number of emergency presentations among older adolescents (aged 16-19 years) in England has increased by $317 \%$ over the past decade, ${ }^{2}$ and now account for $36.3 \%$ of accident and emergency department (A\&E) attendances and almost $20 \%$ of inpatient care. ${ }^{3,4}$

Young adults and adolescents (YAAs) are a vulnerable patient group and mortality has increased over the same period to the point where deaths in patients aged 10-19 years are now greater than in those aged 1-4 years. ${ }^{5}$ The healthcare outcomes for patients aged 10-24 in the UK are now among the worst in Europe. ${ }^{6}$ These striking changes in epidemiology have occurred despite several major governmental initiatives between 1999 and 2009 to reduce inequalities in health status and risk factors in young people. ${ }^{7}$

Authors: ${ }^{A}$ consultant physician and clinical director, Acute Medical Unit, Portsmouth Hospitals NHS Trust, Portsmouth, UK; ${ }^{\mathrm{B}}$ consultant physician and senior clinical research lead, Northwest London CLAHRC, Chelsea and Westminster Hospital, London, UK
It could be argued that acute medical units (AMUs) are protected from this increasing demand, as many acute presentations in YAAs are the result of injuries, drug and alcohol misuse, and exacerbations of highly specialist 'paediatric' conditions, such as cystic fibrosis. However, studies have shown that the rate of inpatient activity for general medicine has also substantially increased, ${ }^{2,8}$ suggesting that AMUs are not immune from these trends. This paper explores the challenges posed by caring for YAAs presenting with acute medical problems and makes suggestions as to how AMUs could better meet these.

\section{How do YAAs present to the AMU?}

There is a lack of precision in the epidemiological data with regard to acute presentations in 15-30 year olds. However, a number of patterns of presentation are reasonably clear:

$>$ new presentations of acute disease - particularly gastrointestinal, endocrine and respiratory illnesses ${ }^{2}$

$>$ new presentations of chronic disease $-25 \%$ of cancer diagnoses now occur in the acute setting ${ }^{9}$

$>$ as a result of risk-taking behaviour, including drug and substance abuse, self-harm and unsafe $\operatorname{sex}^{8}$

$>$ less frequent presentations of patients with chronic, complex disease, experiencing either exacerbations of disease or other intercurrent illnesses, including patients with 'paediatric' conditions, such as cystic fibrosis, transitioning into adult care ${ }^{7}$ $>$ patients with severe and complex disabilities.

The differences in patterns between YAAs and other age groups are partially explained by their strong preference for using acute services rather than seeing a general practitioner. ${ }^{10}$

\section{YAAs and the AMU: challenges}

Caring for adolescents and young adults presents unique challenges in the AMU setting, due to their distinct biological and psychological profiles. YAAs are very different from both children aged $<15$ and adults aged $>25$. Between the ages of 15 and 20 , the process of physiological maturation is still being completed, with continuing endocrine changes and fluctuating hormonal drives. Complex neuropsychological changes are ongoing with, for example, the maturation of the prefrontal cortex; this is involved in inhibitory control, planning and decision-making, and is not completed until late in the third decade, meaning that YAAs may react differently from older 
adults or children in any given situation. ${ }^{11}$ YAAs can also be considered culturally distinct, with their own rules for social interaction, and different interpretations from adults, particularly for subconscious aspects of communication, such as dress, eye contact, body language, and tone and pitch of voice. ${ }^{12}$ The physical, emotional and cultural aspects of maturation also do not necessarily proceed at the same rate, with levels of physical maturation often outstripping emotional and/or social maturation, particularly in males.

These complex developmental changes may impact directly on presentations of illness. Not only are YAAs more likely to present with illnesses and consequences of risk-seeking behaviour, but those with chronic illnesses are less likely to adhere to treatment regimens and disengage from medical care. ${ }^{13}$ This is reflected by the fact that the mortality for patients after renal transplantation is higher in 18-25 year olds than any other group, ${ }^{14}$ with similar findings for patients with diabetes mellitus aged $15-35 .^{15}$

The AMU setting also poses distinct challenges. In the first instance, there is no universal agreement on what the division between 'paediatric' and 'adult' care is or should be. This results in a lack of uniformity not only between hospitals, but also between departments within hospitals. For example, a patient seen in a paediatric A\&E catering for YAAs aged up to 18 may be admitted to an adult ward if the threshold for a paediatric admission is 16 years. Decisions about the most appropriate bed location should take into account a patient's physical and emotional maturation, as well his or her medical needs, but these may not be clear or well understood at the time of admission. Services, such as Childhood and Adolescent Mental Health Services (CAMHSs), may be available in A\&E, but not on the ward. These issues around boundaries of care can lead to discontinuity, fragmentation and delays in care at a time of high medical risk and marked family stress.

The physical environment of the AMU can also be problematic. YAAs value privacy highly, and have a strong preference for personal bathrooms, which are usually in short supply. YAAs also frequently cite the lack of communal space and restricted access to mobile phones and the internet as key problems. ${ }^{16}$ They often have different sleep patterns from adults, which can make the routine of the ward challenging. ${ }^{17}$ AMUs also often have problems providing overnight accommodation for parents and carers for YAAs with chronic/ complex needs, which can be a source of frustration.

Staff in the acute setting often feel that they lack the skills to deal properly with YAAs and this is reflected in the reporting of their symptoms, particularly pain, being much less well managed than in adults. ${ }^{3,18}$ Medical staff report being less confident, not only in caring for unfamiliar conditions, but also in dealing with 'adult' conditions, such as inflammatory bowel disease, in younger patients. ${ }^{19}$ Nurses report feeling poorly prepared to deal with patients with complex needs ${ }^{20}$ and to cope with patients who may have multiple social issues and exhibit challenging or disruptive behaviour on the ward. ${ }^{21}$

Both staff and YAA patients alike report problems with communication in the acute setting. Studies have found that a significant proportion of doctors feel uncomfortable talking to young people. ${ }^{12}$ YAAs are more likely to report lower scores than other adults for being treated with respect and dignity, and rate the skills/attitudes of their doctors less highly. This is especially true of younger females. ${ }^{3}$ This may be attributed, in part, to YAAs reacting more emotionally and less rationally than adults in a similar situation, as well as a lack of staff training in appropriate communication skills.

These issues are all highlighted in patients with chronic conditions who are 'transitioning' from paediatric to adult care. ${ }^{22}$ Where previously their care will have been provided by a stable team with whom they had a long-term relationship, they may be confronted by staff who not only are unknown to them, but may have limited experience in managing their condition. Pathways for care that were previously clear may need renegotiation, with resulting confusion and frustration.

\section{National policy on YAAs}

The comparatively poor outcomes for YAAs have increasingly been recognised at the national level. ${ }^{4,7}$ Not only has the need for better transition of YAAs with chronic or complex conditions from paediatric services been highlighted, but it is recognised that all YAAs should be receiving better and more appropriate care, regardless of the setting. This is reflected in commissioners incorporating ideal standards of care specifically for YAAs within specialist services, such as the Diabetes Best Practice Tariff. More generally, the Department of Health published the You're Welcome quality criteria in $2011 .^{23}$ These have since been endorsed by the World Health Organization. The domains (Table 1) contain recommendations that may be challenging for AMUs, but are likely to be co-opted into standards for YAA care and used to support negotiations with commissioning bodies. The Royal College of Physicians also recently released a position statement on the care of YAAs (Box 1).

\section{Box 1. RCP's position statement on the care of} adolescent and young adult patients

The RCP is committed to the following

$>$ High-quality, developmentally appropriate care, tailored to reflect the unique needs of patients during adolescence and young adulthood.

$>$ A joined-up experience of care, whether patients are accessing acute episodic care or transitioning from paediatric to adult services in the management of long term conditions.

Improving adult physicians' awareness and understanding of how to deliver high-quality, developmentally appropriate care for these patients as normal practice.

Influencing policy-makers, commissioners and providers at all levels to improve the quality of care for young adults and adolescents.

$>$ Listening to young adult and adolescent patients and their parents/carers, and increasing their representation in the RCP's patient involvement activity.

> Advocating for further research to improve our understanding of young adults' and adolescents' health, their experiences of care and their health outcomes.

$\mathrm{RCP}=$ Royal College of Physicians 
Table 1. You're Welcome quality criteria for young person-friendly health services and implications for the acute medical unit (adapted with permission) ${ }^{10,22}$

Access

Publicity

Confidentiality and consent

Environment

Staff training, skills, attitudes and values

Joined-up working

Involvement in monitoring and evaluation of patient experience

Health issues for young people
Outlines how to ensure that services are accessible to young people.

> YAAs should be able to express preferences about who is present during consultations.

$>$ Opportunities should be offered to talk without parents/carers present.

$>$ Clear lines of referral to other services.

Highlights the importance of effective publicity in raising awareness of the services available and explaining the extent of confidentiality.

Addresses confidentiality, consent and safeguarding, and how these are implemented by staff and understood by service users.

$>$ Written policies should be in place with regard to these issues.

$>$ Staff should receive training.

$>$ Patients should receive explicit information.

Addresses service provision, environment and atmosphere, with the aim of ensuring that they are young people friendly (at the same time as being welcoming to all service users, regardless of age).

$>$ Care should be provided in an appropriate environment.

$>$ Pain relief should be optimised.

$>$ Provision should be made for parents/carers.

Addresses the training, skills, attitudes and values that staff need to deliver young person-friendly services.

> All staff should receive appropriate training on understanding and communicating with YAAs.

> Appropriate appraisal, supervision and support should be provided to staff regularly caring for YAAs.

Addresses some of the ways to ensure effective joined-up delivery.

$>$ Relevant services should be co-located where possible.

> Pathways for service delivery and referrals should be clear.

Addresses the importance of young people's involvement in service development, monitoring and evaluation.

Outlines the health needs of young people as they go through the transition into adulthood.

$>$ Consultations should provide opportunities to express concerns about lifestyle issues, such as substance misuse, mental or emotional concerns, and sexual health.

> Pathways for referral for YAAs with emotional and mental health concerns, including to specialised CAMHS, should be clear.

$>$ Care for patients is appropriate to their chronological age and cognitive ability.

CAMHS = childhood and adolescent mental health services; YAAs = young adults and adolescents

\section{Ideal solutions}

Adolescent units have been suggested as the gold standard in caring for adolescents and younger adults (14-19 year olds). Although seemingly suitable for only larger institutions, calculations have found that any hospital with a minimum catchment of 50,000 people would have sufficient numbers of patients to support a 12-bedded adolescent unit. ${ }^{24}$ Co-design projects with YAA contributors favour a combination of fourbed and single rooms, adequate bathroom facilities, and a room for socialising and leisure activities. Staff on adolescent units should receive specialised training and, crucially, have a CAMHS worker on-site each day. Models of consultant cover and responsibility vary, but these could include 'shared care' arrangements, with acute physicians providing input as appropriate. There is little doubt that such units improve patient experience - although only about $10 \%$ of YAAs receive care on such units, patients report receiving much better care than in either child or adult settings. ${ }^{25}$

Where adolescent units are not feasible, some of this gap could potentially be filled by a young persons team. As a minimum, this could consist of a lead nurse and CAMHS or mental health liaison workers, but could also include or link with: nurse specialists in learning disabilities, and substance misuse or sexual health; physiotherapists; social workers; and nutritionists. As well as providing stable outpatient services, these teams are able to support acute admissions by ensuring that the environment is as suitable as possible, supporting staff with training, and providing some degree of continuity of care when the patient is transferred to another team or is discharged. 


\section{Practical tips for the AMU}

Although redesigning services specifically to accommodate YAAs might seem onerous and expensive, there is still much that can be done quickly and easily to improve the care that adolescents and young adults receive on AMUs. Aspects of care that might be rapidly addressed include:

$>$ having clear pathways for the acute care of those aged between 14 and 19 years. This will require discussion by A\&E, the AMU and paediatric services to locally agree chronological and/or developmental criteria for the place of inpatient care

$>$ being aware of the support services available for YAAs. This should include pathways of referral to CAHMS, if available

$>$ appointing a lead clinician and lead nurse to act as champions, ensuring that the needs of YAAs are considered in all aspects of AMU design/operation/training

$>$ ensuring that patient information leaflets are appropriate for younger patients, as well as providing leaflets on accessing age-appropriate health support services

$>$ policies covering visits and/or accommodation of patients and carers should be reviewed and revised with younger patients with complex needs in mind

$>$ ensuring that young patients have adequate pain relief.

Staff should receive basic training on communicating with younger patients. Tips for improving communication are outlined in Box 2.

\section{Box 2. Tips for dealing with young adults and adolescents}

Show respect for privacy of a young patient, who may consider a closed curtain to be the equivalent of a locked door.

$>$ Be seated at the same level, whenever possible, and use open body language.

Establish rapport before starting clinical discussions.

Young patients are particularly insistent that they be regarded as separate from their disease; terms such as 'asthmatic' and 'diabetic' are best avoided.

> Be prepared to explain concepts at length and in a consistent manner.

Offer choice whenever possible, even if no choice is really available, eg 'You need to stay in hospital - would you prefer your mum to be with you?'

> Use 'then and when' statements, eg 'When you have had your blood test, then you can go off for a coffee.'

Do not make promises that cannot be delivered, especially about investigations and discharge.

> Show respect for confidentiality, this needs to be addressed early and may require an explicit discussion about what will or will not be discussed with parents/carers/other professionals.

$>$ Offer time for discussion without parents/carers; this should be done routinely.

>ffer time to discuss other health concerns, such as substance misuse, sexual health, and emotional and psychological concerns.

\section{Bridging the gap between paediatric and adult} services

Establishing better relationships between the AMU and paediatric services could also make an immediate and concrete difference to patient care. Many paediatric teams now actively 'transition' patients from paediatric to adult services, ${ }^{22}$ but there is frequently a gap concerning what happens to patients who require emergency adult admission during the transition process. Being aware of how local transition programmes function can, in itself, be extremely useful, because many have identified team leads, records of care plans and agreed pathways for follow-up. AMUs can also look to take a more active role in transition programmes, particularly in bigger units where there may be scope to identify an AMU lead for YAAs. This might include helping to arrange visits for YAAs to the AMU, agreeing advance plans for acute care in discussion with patients and paediatric staff, or working on shared care pathways for transitioning patients.

Where there is no local paediatric service or no transitional programmes are offered, many of the key principles are still applicable. Patients aged 14-19 years who are frequent attenders or have complex care needs can be identified. Individual management plans can then be formulated, with input from the responsible paediatrician, and the family and specialist services, before being shared with any involved parties, such as the family, the GP, ambulance services and school/college. Copies can be kept on the AMU or marked on the electronic patient record where available. After each admission, feedback can be sought from the patient and his or her family and the care plan updated as needed. Experience suggests that such care planning can significantly impact on experience, improve care and help prevent complaints.

\section{Conclusion}

YAAs present to AMUs more frequently and are more prone to poor outcomes than previously recognised. By considering the distinct needs of YAAs using nationally agreed templates, such as the You're Welcome criteria, responsive, patient-centred care can be delivered to this vulnerable group. AMUs need to liaise with colleagues in paediatric and emergency medicine, as well as other stakeholders, to streamline and improve care delivery. Although this may present a challenge to many AMUs, changes should benefit patients and staff alike.

\section{References}

1 Viner RM, Barker M. Young people's health: the need for action. BMJ 2005;330:901-3.

2 Hargreaves DS, Viner RM. Adolescent inpatient activity 1999-2010: analysis of English Hospital Episode Statistics data. Arch Dis Child 2013;0:1-4.

3 Hargreaves DS, Viner RM. Children's and young people's experience of the National Health Service in England: a review of national surveys. BMJ 2012;97:661.

4 Department of Health. Achieving equity and excellence for children. London: DH, 2010.

5 Patton GC, Coffey C, Sawyer SM et al. Global patterns of mortality in young people: a systematic analysis of population health data. Lancet 2009;374:881-92. 
6 Viner RM, Hargreaves DS, Coffey C, Patton GC, Wolfe I. Death in young people aged 0-24 years in the UK compared with the EU15+ countries: analysis of WHO Mortality Database. Lancet 2014;384:880-92.

7 Hargreaves DS, Djafari Marbini A, Viner RM. Inequality trends in health and future health risk among English children and young people, 1999-2009. Arch Dis Child 2013;98:850-5.

8 Hagell A, Coleman J, Brooks F. Key data on adolescence 2013. London: Association of Young People's Health, 2013.

9 Purkayastha D, O'Hara, Moran T et al. Routes to diagnosis: investigating the different pathways for cancer referrals in England for teenagers and young adults. London: National Cancer Intelligence Network, 2013.

10 Fortuna RJ, Robbins BW, Mani N, Halterman JS. Dependence on emergency care among young adults in the United States. J Gen Intern Med 2010;25:663-9.

11 Buitelaar JK. Adolescence as a turning point: for better and worse. Eur Child Adolesc Psychiatry 2012;21:357-9.

12 White B, Viner RM. Improving communication with adolescents. Arch Dis Child Educ Pract Ed 2012;97:93-7.

13 McGrady ME, Hommel KA. Medication adherence and health care utilization in pediatric chronic illness: a systematic review. Pediatrics 2013;132:730-40.

14 National Institutes of Health. Five year graft survival probabilities: all deceased donor transplants. In: US Renal Data System 2011 annual data report: atlas of chronic kidney disease \& end-stage renal disease in the United States. Bethesda, MA: National Institutes of Health, 2010.

15 Nakhla M, Daneman D, To T, Paradis G, Guttmann A. Transition to adult care for youths with diabetes mellitus: findings from a universal health care system. Paediatrics 2009;124:e1134.

16 Payne D, Kennedy A, Kretzer V, Turner E, Shannon P, Viner R. Developing and running an adolescent inpatient ward. Arch Dis Child Educ Pract Ed 2012;97:42-7.
17 Wiggins SA, Freeman JL. Understanding sleep during adolescence. Pediatr Nurs 2014;40:91-8

18 Hargreaves DS, Sizmur S, Viner RM. Do young and older adults have different health care priorities? Evidence from a national survey of English inpatients. J Adolesc Health 2012;51:528-32.

19 Sebastian SI, Jenkins H, McCartney S et al. The requirements and barriers to successful transition of adolescents with inflammatory bowel disease: differing perceptions from a survey of adult and paediatric gastroenterologists. J Crohn's Colitis 2012;6:830-44.

20 Law J, McCann D, O'May F, Smart C, Buchan J. Service provision for children and young people with complex needs in a community setting from the perspectives of nursing and allied health professionals. Edinburgh: Scottish Government Social Research, 2009.

21 Rutledge DN, Wickman ME, Cacciata $\mathrm{M}$ et al. Hospital staff nurse perceptions of competency to care for patients with psychiatric or behavioral health concerns. J Nurses Prof Dev 2013;29:255-62.

22 Gleeson H, McCartney S, Lidstone V. 'Everybody's business': transition and the role of adult physicians. Clin Med 2012;12:561-6.

23 Department of Health. You're Welcome quality criteria: making health services for young people friendly. London: DH, 2011.

24 Viner RM. National survey of use of hospital beds by adolescents aged 12 to 19 in the United Kingdom. BMJ 2001;322:957-8.

25 Hargreaves DS, McDonagh JE, Viner RM. Validation of You're Welcome quality criteria for adolescent health services using data from national inpatient surveys in England. J Adolesc Health 2013;52:50-7.

Address for correspondence: Dr L Vaughan,

Northwest London CLAHRC, 4th Floor, Lift Bank D,

Chelsea and Westminster Hospital, 369 Fulham Rd,

London SW10 9NH, UK.

Email: louella.vaughan@chelwest.nhs.uk

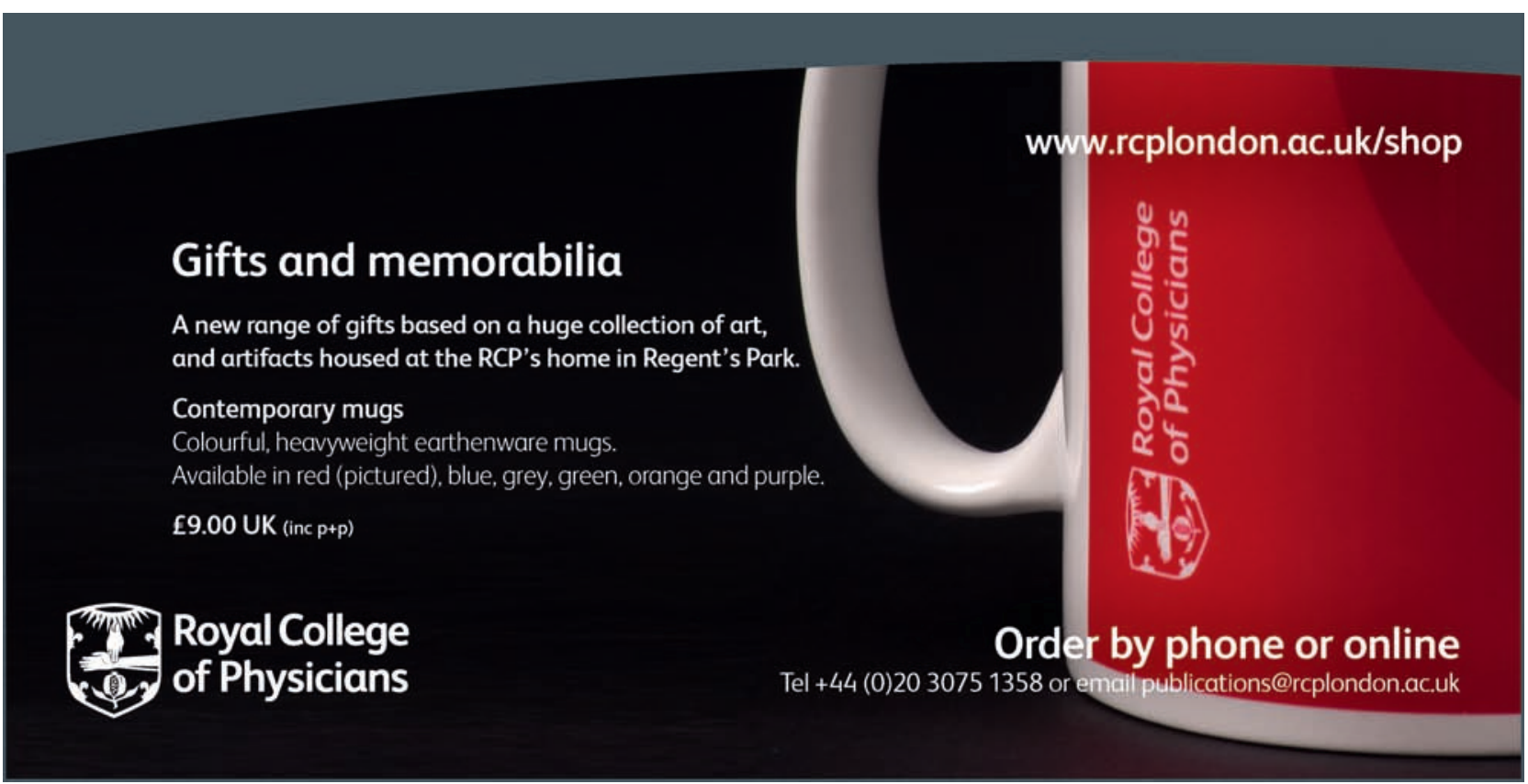

